\title{
A PROPOSTA DE UMA TEORIA GERAL DE PRINCÍPIOS DE ABSTRAÇÁO: UMA CONTRIBUIÇÃO À FUNDAMENTAÇÁO DA ARITMÉTICA
}

\author{
André Nascimento Pontes
}

\begin{abstract}
RESUMO: O objetivo do presente artigo é apresentar a correlação entre o programa logicista fregeano de fundamentação da aritmética, o neologicismo de Crispin Wright e os chamados princípios de abstração. Minha tese é que uma análise geral de princípios de abstração, do ponto de vista lógico e explanatório, é mais basilar que o projeto de fundamentação da aritmética na forma como ele foi proposto pelo logicismo. Isso fica evidente através do fato de que o fracasso do programa logicista esteve intimamente ligado à imprecisão e inconsistência no uso de procedimento de abstração.
\end{abstract}

PALAVRAS-CHAVE: Lógica; Filosofia da Matemática; Aritmética; Princípio de Abstração.

Em linhas gerais, princípios de abstração constituem mecanismos lógico-semânticos que operam com a função de introduzir o discurso acerca de objetos abstratos em uma teoria - no caso específico da aritmética, onde se concentram os esforços deste artigo, a introdução do discurso a respeito de números cardinais - juntamente com critérios de identidade que tais objetos devem obedecer ${ }^{2}$. Diante da centralidade do uso de instâncias do princípio de abstração na realização de importantes programas filosóficos e nas propostas de distinçôes conceituais básicas desenvolvidas nos campos da Metafísica, Epistemologia, Filosofia da Matemática, dentre outros, penso que a tentativa de realizar uma análise geral de tais princípios configura um projeto de extrema relevância para os fundamentos da própria atividade filosófica. Nesse

\footnotetext{
${ }^{1}$ Professor Assistente do Departamento de Filosofia da Universidade Federal do Amazonas (UFAM) e Doutorando em Filosofia pela Pontifícia Universidade Católica do Rio de Janeiro (PUC Rio). E-mail: philospontes@gmail.com

${ }^{2}$ Ao longo do texto, usarei o termo "princípios de abstraçáo" (no plural) para me referir às mais diferentes instâncias de um esquema proposicional geral que é utilizado para obter definiçôes contextuais ou parciais a partir das quais introduzimos entidades abstratas em nossas teorias. Uma fomalizaçáo desse esquema será apresentada na Seção 1. Nesse contexto, usarei o termo "princípio de abstração" (no singular) para me referir a esse esquema proposicional.
} 
sentido, a análise de princípios de abstração possui um caráter metafilosófico e conexôes claras não apenas com problemas técnicos formulados em Filosofia da Matemática, mas também com questôes filosóficas mais amplas. Por exemplo, Aristóteles já fazia uso de procedimentos de abstração para explicar nosso conhecimento sobre universais enquanto classe de objetos semelhantes. Partindo desse contexto geral, parece natural pensar que os resultados de uma teoria de princípios de abstração podem oferecer os recursos básicos de análise de questóes em diversos campos da pesquisa filosófica. Um exemplo relevante seria a distinção entre os termos "abstrato" e "concreto" - de extrema importância para o debate acerca dos universais - que só pode ser devidamente formulada com base em uma explanação da constituição e dos critérios de aceitabilidade do que entendemos por objetos abstratos.

No presente artigo, concentro meus esforços na tentativa de apresentar a necessidade de uma teoria geral de princípios de abstração, tomando como contexto argumentativo as conexóes entre procedimentos de abstração e a Filosofia da Matemática. Meu ponto de partida é a discussão da relação de tais princípios com a execução do programa logicista de Frege - e isso inclui sua posição no fracasso desse mesmo programa - bem como com a formulação das alternativas neologicistas desenvolvidas nas últimas décadas. A tese básica que orienta meu artigo é que a realização de uma teoria geral de princípios de abstração é, de um ponto de vista lógico e filosófico, mais fundamental que a própria execução da fundamentação da aritmética tomada do quadro teórico do logicismo. A justificativa para tal afirmação é que, somente a partir do momento no qual posso oferecer critérios sólidos para identificar uma boa instância do princípio de abstração - ou seja, uma instância livre de paradoxos -, é que posso garantir a consistência do conjunto de resultados que derivamos a partir dela.

O programa logicista em Filosofia da Matemática pode ser caracterizado, em linhas gerais, como a proposta de redução de todas as proposiçôes básicas da aritmética a proposiçóes da lógica. A tese central do logicismo é mostrar não só que, em última instância, a aritmética pode ser expressa em termos estritamente lógicos, mas, também, de maneira mais fundamental, que as leis básicas da aritmética podem ser derivadas de leis lógicas. ${ }^{3}$ É fato notório que

${ }^{3}$ De acordo com Quine, o programa de redução em fundamentos da matemática se dá em duas direçôes: por um lado, há uma ideia de uma reduçâo conceitual - no caso específico do logicismo, isso equivale à tentativa de expressar todas as noçôes aritméticas em termos estritamente lógicos. Mas isso não garante ainda que a lógica é mais fundamental que a aritmética. Por isso, é imprescindível um segundo passo, que Quine chama de redução doutrinal, através do qual todas as leis aritméticas possam 
o fracasso do programa logicista de fundamentação da aritmética, na versão proposta por Frege, deve-se fundamentalmente à formulação do Paradoxo de Russell, a partir do polêmico Axioma $V$ utilizado, dentre outras coisas, para provar o chamado Princípio de Hume; ambos, instâncias do princípio de abstração. Muitos filósofos e matemáticos apontam tal acontecimento tanto como um marco na crise dos fundamentos, que inspirou avanços sem precedentes na Filosofia da Matemática, quanto o ponto de origem do que chamamos de teoria axiomática dos conjuntos.

A despeito do fracasso do programa fregeano de fundamentação da aritmética, as últimas décadas foram palco de propostas de revisão das principais etapas de execução do programa de Frege, tomando como base alguns resultados técnicos recentes obtidos em lógica e filosofia da matemática. O objetivo principal era oferecer uma versão consistente do ideal de redução da aritmética à lógica. ${ }^{4}$ Tais propostas são comumente denominadas na literatura como neologicistas. Em geral, como veremos adiante, as propostas neologicistas mais influentes se concentram na tentativa de mostrar que é possível oferecer uma definiçáo relevante de número cardinal sem apelar para noção de extensóes de conceitos, a exemplo do que fez Frege, ao utilizar o Axioma V. Para alguns neologicistas, uma nova definição deveria ser introduzida contextualmente, através do Princípio de Hume. Se isso for possível, podemos oferecer uma versão do logicismo onde o Axioma $\mathrm{V}$ é dispensável e, consequentemente, onde o paradoxo de Russell não pode ser derivado. Nesse contexto, fica claro que o passo fundamental para o neologicista seria mostrar a validade do Princípio de Hume a ponto de legitimar seu uso como um axioma dentro do sistema.

Da forma como planejei o presente artigo, ele se encontra divido em três seções: na Seção 1, apresento de forma panorâmica a relevância do Axioma V e do Princípio de Hume para a execução do logicismo de Frege, bem como a relação desses princípios com o que chamarei de esquema geral de abstração. A Seção 2 é dedicada a uma apresentação dos principais traços da proposta neologicista de Crispin Wright. Por fim, na Seção 3, tento demonstrar que o sucesso da proposta de Wright - se ele é possível - depende, em grande parte,

ser derivadas a partir de leis estritamente lógicas. A redução conceitual se preocupa com o significado e a expressabilidade de uma teoria, com base nos conceitos de outra, ao passo que uma redução doutrinal se preocupa com a verdade e a derivabilidade de uma teoria, através de outra mais fundamental. Cf. QUINE, W. v. O. Naturalized Epistemology. In: Ontological Relativity and Others Essays. New York: Columbia University Press, 1969.

${ }^{4}$ Dentre os trabalhos técnicos mais influentes, estão: WRIGHT (1983), BOOLOS (1987) e HECK (1993). 
de uma análise geral do estatuto lógico-semântico de princípios de abstração. Penso que, embora posteriormente tal análise se revele insuficiente para o sucesso do programa neologicista, dela podemos ainda derivar resultados filosóficos relevantes envolvendo aspectos ontológicos e epistemológicos de objetos abstratos.

\section{O princípio de abSTração e o logicismo de Frege}

Embora procedimentos de abstração estejam presentes desde a tradição clássica, por exemplo, no processo de derivação de universais a partir de classe de objetos semelhantes na metafísica aristotélica, foi Frege o primeiro a oferecer um estudo formal, claro e convincente que correlaciona o uso de princípios de abstração à constituição de uma ontologia de objetos abstratos. É fato notório que o interesse fundamental de Frege era a demonstraçáo do seu programa logicista e, por isso, sua análise de procedimentos de abstração se concentrava, em última instância, na derivação de um tipo especial de objeto abstrato, a saber, números cardinais. A estreita relação do programa filosófico de Frege e o uso de procedimentos de abstração fica evidente, como veremos a seguir, através da centralidade do uso de duas instâncias do princípio de abstração na execução e fracasso do logicismo, a saber, o axioma V e o princípio de Hume.

O núcleo do programa logicista fregeano está centrado no problema da explanação da estrutura dos objetos descritos pela aritmética básica, ou seja, os números, - que, para Frege, possuem natureza lógica - bem como na forma como esses números são dados a nós. Contra Kant, que defendeu a natureza sintética a priori do conhecimento matemático - e isso inclui tanto a geometria quanto a aritmética - Frege defendeu a tese de que as proposiçóes da aritmética são analíticas no sentido dado nos Fundamentos da Aritmética $\$ 3$, isto é, prováveis exclusivamente com base em leis lógicas juntamente com definiçóes relevantes. Da forma como interpreto a questão, a execução do programa desenvolvido por Frege passa por dois pontos preliminares: (1) pelo estabelecimento de uma linguagem formal através da qual todas as proposiçôes da aritmética possam ser expressas; e esse foi precisamente o projeto realizado com sucesso por Frege, em sua Begriffsschrift, onde ele expôs a base da lógica de predicados; e (2) pela apresentação de definiçôes básicas que ofereçam de forma clara critérios de identidade para introduzir os objetos que o domínio matemático pressupóe; e isso, como penso, passa pela tentativa de oferecer uma explanaçáo adequada para nosso conhecimento de objetos abstratos - no 
caso específico da aritmética, o conhecimento sobre números cardinais - via análise de princípios de abstração. $\mathrm{O}$ núcleo do presente artigo se concentra na relação entre a formulação de uma teoria geral de princípios de abstração e a execução do tópico (2) mencionado anteriormente.

De acordo com Frege, toda asserção numérica constitui uma afirmação sobre predicados ou conceitos. Por exemplo, dizer que "quatro era o número de músicos dos Beatles" equivale a dizer que o predicado ou conceito ser músico dos Beatles tem a propriedade de possuir quatro objetos que caem sob ele. A questão era como realizar a passagem das afirmaçóes sobre propriedades numéricas para os números propriamente ditos. Para isso, Frege introduziu o chamado Princípio de Hume, em que podemos encontrar algo como um "operador de cardinalidade" ("NxFx", ou seja, o número que convém ao conceito F). De maneira formal, o Princípio de Hume pode ser enunciado como segue:

\section{$\mathrm{NxFx}=\mathrm{NxGx} \leftrightarrow \mathrm{F} \approx \mathrm{G}$}

onde o símbolo “ $\approx$ " denota a correspondência biunívoca entre os elementos que entram na composição das extensóes de F e G. O que a formalização fregeana do Princípio de Hume afirma basicamente é que o número de $F s$ é o mesmo que o número de $G s$ se, e somente se, houver uma correspondência biunívoca (um-a-um) entre os objetos que são $F_{s}$ e os objetos que são $G s$. De modo análogo, Frege oferece um critério claro para definir equinumerosidade, a saber, os conceitos $\mathrm{F}$ e $\mathrm{G}$ são equinuméricos se, e somente se, existe uma correspondência biunívoca entre os elementos que compóem a extensão de $\mathrm{F}$ e os elementos da extensão de $\mathrm{G}$, isto é, uma correspondência um-a-um entre os membros dos conjuntos de Fs e Gs. É importante notar que o Princípio de Hume introduz na formulação do programa logicista fregeano um novo tipo de objeto, a saber, números cardinais. Ele opera enquanto uma definição contextual, tendo em vista que sua função é estabelecer as condiçôes de verdade de toda sentença envolvendo atribuições numéricas.

Embora o Princípio de Hume, juntamente com a lógica de segunda ordem, ofereça uma base consistente para o tratamento formal dos axiomas da aritmética de Peano, a princípio, ele não constitui uma verdade estritamente lógica e, portanto, não pode ser utilizado como base para o programa logicista. Para além dessa objeção, Frege tinha percebido (FREGE, 1983, \$\$66-67) o fato de que, tomado isoladamente, o Princípio de Hume não possibilitava dar

${ }^{5}$ Cf. Os Fundamentos da Aritmética, $\$ \$ 60-68$. 
uma solução para o que ficou conhecido na literatura como o Problema Júlio César, a saber, como identificar e diferenciar os números de outros tipos de objetos. ${ }^{6} \mathrm{~A}$ ideia é que o Princípio de Hume oferece um recurso elegante para verificar o valor de verdade de uma afirmação da forma

\section{$\mathrm{NxFx}=\mathrm{NxGx}$}

ou seja, para verificar as condiçóes de verdade da afirmação de que a dois conceitos convém o mesmo número. No entanto, ele falha em apresentar as condições de verdade para uma sentença da forma

$$
a=\mathrm{NxFx}
$$

a qual sustenta ser $a$ o número que convém ao conceito F. Tomando o exemplo oferecido por Frege: como saber que Júlio César não é o número que convém a um conceito qualquer $\mathrm{j}$ ? Ou ainda, como saber se é verdade que $\exists \varphi($ Júlio César $=\mathrm{Nx} \varphi \mathrm{x})$ ?

Como uma solução para esse impasse, Frege introduziu o Axioma V como um recurso para, a uma só vez, provar a validade do Princípio de Hume e também prover o meio para uma definição explícita de número cardinal em termos de extensôes de conceitos que eliminasse o Problema Júlio César ${ }^{7}$.

Grosso modo, o Axioma V de Frege pode ser formulado como segue:

$$
\{\mathrm{x}: \mathrm{Fx}\}=\{\mathrm{x}: \mathrm{Gx}\} \equiv \forall \mathrm{x}(\mathrm{Fx} \leftrightarrow \mathrm{Gx})
$$

o que, em linguagem natural, equivale à seguinte afirmação: dizer que dois conjuntos $\mathrm{F}$ e $\mathrm{G}$ são idênticos é o mesmo que dizer que $\mathrm{F}$ e $\mathrm{G}$ possuem a mesma extensão, ou seja, os objetos $F s$ e os objetos $G s$ são precisamente os mesmos objetos. No Axioma V, os termos do tipo " $\{\mathrm{x}: \mathrm{Fx}\}$ ” funcionam como um operador de abstração que permite falar sobre a extensão de um conceito $\mathrm{F}$ qualquer. Em linhas gerais, o Axioma V será usado para introduzir uma definição de números enquanto classes de extensôes de conceitos equinuméricos ${ }^{8}$. Respondendo de maneira simplificada ao problema anterior:

\footnotetext{
${ }^{6} \mathrm{O}$ problema ganhou o nome esdrúxulo de Problema Júlio César, pois Frege o apresentou através da questáo: "[como saber] se a um conceito convém o número Júlio César, ou ainda, se este famoso conquistador da Gália, é ou não um número?” Cf. Os Fundamentos da Aritmética, \$56. Em última instância, o que está em jogo, na formulação do problema Júlio César, é a busca por um critério de aplicação para números que determine a que tipos de conceitos um objeto $x$ deve estar associado para que $x$ seja um número.

${ }^{7}$ Para uma introdução ao estudo do Axioma V de Frege, cf. HECK (1998).

${ }^{8}$ Vale ressaltar que, para Frege, extensões são também objetos.
} 
tendo em vista que Júlio César não é uma extensão, então ele também não é um número e, portanto, estaria solucionado o Problema Júlio César?.

$\mathrm{O}$ que está por trás tanto da formulação do Axioma $\mathrm{V}$ quanto do Princípio de Hume - e que constitui o núcleo de interesse do presente artigo são os chamados princípios de abstração. O Axioma V e o Princípio de Hume são instâncias do princípio de abstração que Frege usa com o objetivo de introduzir objetos abstratos - a saber, números cardinais -, em sua teoria, juntamente com critérios de identidade bem definidos para esses mesmos objetos. ${ }^{10} \mathrm{De}$ fato, a função central dos princípios de abstração deve ser (ou deveria ser) a de estabelecer os critérios de identidade que possibilitem introduzir de forma legítima e segura o discurso sobre objetos abstratos. Esse é, por exemplo, o caso do Princípio de Hume no logicismo fregeano, o qual permite gerar o discurso sobre números cardinais a partir de noçóes mais básicas, tais como a de correlação biunívoca (correspondência um-a-um) ou a de equinumerosidade.

Em linhas gerais, as diferentes instâncias do princípio de abstração são nada mais que casos específicos de um esquema geral de abstração com a seguinte forma:

$$
\forall \mathrm{x} \forall \mathrm{y}\left(\sum \mathrm{x}=\Sigma \mathrm{y} \leftrightarrow \mathrm{x} \approx \mathrm{y}\right)
$$

onde " $\Sigma$ " é um operador aplicável a expressões do tipo das variáveis "x" e "y" - um exemplo pode ser termos singulares - e "ح” é uma relação de equivalência entre entidades denotadas pelos termos que substituem as variáveis " $x$ " e " $y$ ". No caso específico em que as variáveis " $x$ " e "y" variam sobre objetos, o termo " $\approx$ ” ocorre enquanto uma relaçáo diádica de primeira ordem, e o princípio de abstração resultante representa uma abstração de primeira ordem sobre objetos. De maneira análoga, tanto o Axioma V quanto

\footnotetext{
${ }^{9}$ É claro que, em uma análise mais rigorosa do Problema Júlio César, é sempre possível levantar a questão "como sabemos que Júlio César não é uma extensão?" A crítica-padrão a essa solução ingênua é que, em última instância, ela apenas transfere o problema: se, de início, a questão era oferecer um critério que determinasse se Júlio César é ou não um número, agora o problema é oferecer um critério que determine se Júlio César é ou não uma extensão. Ao que parece, nos Fundamentos da Aritmética, Frege tomava a noção de extensão de um conceito como fundamental e não problemática.

${ }^{10}$ Para uma análise introdutória da noção de "objeto abstrato", cf. LOWE, E. J. The metaphysics of abstracts objects. The Journal of Philosophy, v. XCII, n. 10, out. de 1995. Lowe apresenta basicamente três concepções de objeto abstrato: um objeto pode ser entendido como abstrato (1) enquanto oposto ao concreto, ou seja, não localizado no espaço-tempo e destituído de interação causal com objetos concretos, (2) enquanto algo concebido como incapaz de desfrutar de uma existência independente e, por fim, (3) enquanto algo que é concebido e introduzido através de princípios de abstração. É precisamente esse terceiro sentido de abstração que está em questão, no presente trabalho.
} 
o Princípio de Hume podem ser apresentados como instâncias de ordem superior do esquema geral de abstraçáo com base nas quais o conceito de número cardinal pode ser explanado. No caso específico do Princípio de Hume, a tese de que "o número de $F$ s é o mesmo que o número de Gs se, e somente se, houver uma correspondência biunívoca entre os $F_{s}$ e os Gs", é formalmente equivalente a um princípio de abstração de ordem superior, no qual o termo " $\approx$ " expressa a equivalência entre extensóes de conceitos - no caso específico, os conceitos $F$ e $G$.

O que Russell mostrou, e que causou o colapso do programa logicista de Frege, foi que o uso irrestrito de definiçóes por abstração pode gerar situaçóes paradoxais que colocam em xeque a consistência do sistema que as utiliza. O exemplo clássico derivado dentro programa fregeano é o seguinte: através do paradoxo que leva seu nome, Russell mostrou que, em última instância, o Axioma V utilizado por Frege - que, como vimos, é uma instância do esquema geral de abstração - implica que, correspondendo a toda propriedade de entidades matemáticas, existe um conjunto de entidades matemáticas que possuem aquela propriedade. ${ }^{11}$ Logo, tomando a propriedade definida dentro da teoria ingênua dos conjuntos, "o conjunto que não contém a si mesmo como elemento", é fácil observar que tal propriedade pode ser usada para expressar uma classe ou conjunto $\mathrm{F}$, que possui como membros apenas os conjuntos que não contêm a si mesmos como elemento. A situaçáo paradoxal surge quando perguntamos se $\mathrm{F}$ é ou não um membro de $\mathrm{F}$. A conclusão é que $(\Phi \in \Phi \leftrightarrow \neg(\Phi \in \Phi))$ e que é logicamente equivalente a $((\Phi \in \Phi) \wedge \neg(\Phi \in \Phi))$.

Podemos observar também, como ressaltam Demopoulos e Clark (2007, p. 133), de maneira didática, que, na direção

$$
\forall \mathrm{x}(\mathrm{Fx} \leftrightarrow \mathrm{Gx}) \rightarrow\{\mathrm{x}: \mathrm{Fx}\}=\{\mathrm{x}: \mathrm{Gx}\}
$$

\footnotetext{
${ }^{11}$ De forma um pouco mais detalhada, a ideia é que podemos mostrar que, com base no Axioma $\mathrm{V}-\{\mathrm{x}: \mathrm{Fx}\}=\{\mathrm{x}: \mathrm{Gx}\} \equiv \forall \mathrm{x}(\mathrm{Fx} \leftrightarrow \mathrm{Gx})$-, é possível definir a noção de pertinência (membership), a saber, $\mathrm{x} \in \mathrm{y} \equiv \exists \mathrm{H}(\mathrm{Hx} \wedge \mathrm{y}=\{\mathrm{x}: \mathrm{Hx}\}) . \mathrm{O}$ fato é que, no contexto da lógica de segunda ordem, o Axioma $\mathrm{V}$, juntamente com a presente formulaçáo da noção de pertinência, implica o chamado axioma ingênuo da compreensão (Naive Comprehension Axiom), presente na teoria ingênua dos conjuntos, a saber, $\forall \mathrm{P} \exists \mathrm{z} \forall \mathrm{x}(\mathrm{x} \in \mathrm{z} \equiv \mathrm{Px})$. O que o axioma da compreensão afirma é que, para toda propriedade, há um conjunto que consiste precisamente dos indivíduos que satisfazem a propriedade em questáo. No caso específico onde nenhum indivíduo satisfaz a propriedade, o conjunto associado a essa mesma propriedade consiste no conjunto vazio $(\varnothing)$. Vale ressaltar que resultados recentes (PARSONS, 1987) mostraram que, no contexto da lógica de primeira ordem, qualquer conjunto de instâncias do Axioma V é consistente, ou seja, o fragmento da lógica de primeira ordem do programa logicista fregeano é consistente. O problema é que a lógica de primeira ordem não é potente o suficiente para os interesses do programa logicista.
} 
o Axioma $\mathrm{V}$ apresenta o caráter funcional da relação que associa todo conceito a uma extensão, ao passo que a direção

$$
\{\mathrm{x}: \mathrm{Fx}\}=\{\mathrm{x}: \mathrm{Gx}\} \rightarrow \forall \mathrm{x}(\mathrm{Fx} \leftrightarrow \mathrm{Gx})
$$

mostra que essa mesma função é biunívoca (um-a-um). No entanto, o que o paradoxo de Russell evidenciou é que não existe tal função. Se assumimos que o Axioma $\mathrm{V}$ implica que a totalidade das extensóes de conceitos corresponde ao conjunto potência (power set) do conjunto de objetos que caem sob eles, então o Axioma V contraria o teorema de Cantor, segundo o qual não existe nenhuma correspondência biunívoca entre um conjunto $x$ e o conjunto potência de $x, P(\mathrm{x})$. Em resumo, o que Russell apontou, através do seu paradoxo, é que o programa logicista, da forma que ele foi proposto por Frege, não possui um modelo que o satisfaça ${ }^{12}$.

\section{O PROGRAMA NEOLOGICISTA E O PRINCÍPIO DE ABSTRAÇÁO}

O núcleo das propostas neologicistas se concentra, via de regra, na tentativa de oferecer uma base axiomática que evite formulaçóes paradoxais e a consequente inconsistência do programa de redução da aritmética à lógica (HALE; WRIGHT, 2001). Essa é a ideia básica por trás da estratégia proposta por Crispin Wright, no seu livro Frege's conception of numbers as objects (WRIGHT, 1983). Segundo Wright, o programa fregeano de redução da aritmética de Peano a um conjunto básico de proposiçóes formuladas a partir da lógica de predicados de segunda ordem foi realizado em duas etapas distintas: em um primeiro momento, Frege empregou o inconsistente Axioma V para derivar o Princípio de Hume. No segundo momento, o Princípio de Hume foi usado - juntamente com a lógica de segunda ordem - para derivar as afirmaçôes básicas da aritmética de Peano. Como Wright defende, o Axioma $V$ não é efetivamente utilizado na segunda parte da derivação fregeana. Dessa forma, se for possível comprovar que, ao contrário do Axioma V, o Princípio

\footnotetext{
${ }^{12}$ Após a demonstração do paradoxo, o próprio Russell tentou salvar o programa logicista, formulando uma versão alternativa. Cf. RUSSELL, B.; WHITEHEAD, A. N. Principia Mathematica [1910-1913] 3 vols. Cambridge: Cambridge University Press, 1978. Através dessa tentativa, ele derivou análises relevantes acerca do princípio do círculo vicioso e da noção de impredicatividade que resultaram em sua teoria dos tipos lógicos. Ocorre que, para um novo fracasso do programa logicista, dentre outros problemas, a definição de número proposta por Russell pressupunha, através do que ficou conhecido com o axioma da infinidade, a existência de infinitos objetos no universo. Obviamente, tal axioma não é lógico e sua aceitabilidade é extremamente polêmica. Para uma análise introdutória do logicismo de Russell, cf. GODWYN, M.; IRVINE, A. Bertrand Russell's Logicism. In: GRIFFIN, N. The Cambridge Companion to Bertrand Russell. Cambridge: Cambridge University Press, 2003.
} 
de Hume é consistente com a lógica de segunda ordem, poderíamos então mostrar que a primeira etapa da derivação fregeana é dispensável, eliminando assim o Axioma $\mathrm{V}$ do programa logicista e nos concentrando apenas na segunda etapa da derivação. ${ }^{13}$ Nesse sentido, o Princípio de Hume poderia ser assumido como analítico dentro do programa de redução logicista e usado para obter, não uma definição de número cardinal em termos de extensões de conceitos, mas uma definição contextual estabelecida por estipulação. Em outras palavras, o Princípio de Hume seria usado como um axioma ou uma definição primitiva para obter uma extensão conservativa da lógica de segunda ordem, pela qual o programa de redução da aritmética fosse possível.

Importantes resultados foram apresentados nas últimas décadas, no sentido de demonstrar que o Princípio de Hume é consistente com a lógica de segunda ordem. Nesse contexto, destaca-se Boolos (1987), onde podemos encontrar um esboço de uma teoria formal FA ("Frege Arithmetic") para o programa logicista fregeano, contendo um equivalente do Princípio de Hume que captura a estrutura básica das teses centrais presentes nos Fundamentos da Aritmética (\$\$ 68-83) e para a qual Boolos apresenta uma prova de consistência com a aritmética básica de Peano. Em linhas gerais, a teoria formal FA, proposta por Boolos, é completamente expressa através da lógica de segunda ordem, enquanto uma extensão do sistema apresentado por Frege, na Begriffsschrift. O objetivo de Boolos era mostrar que é possível oferecer um modelo para o Princípio de Hume tanto na teoria dos conjuntos quanto na aritmética de segunda ordem e que, portanto, o Axioma $V$ seria dispensável para a execução do programa logicista.

Embora a relevância desses resultados em torno da consistência lógica do Princípio de Hume, com objetivo de salvar o programa fregeano de redução da aritmética à lógica, seja vista com certa cautela, da mesma forma que Hale e Wright (2007), penso que a credibilidade das propostas neologicistas deve passar invariavelmente por uma análise do estatuto lógico-semântico de princípios como o Axioma V e o Princípio de Hume. Nesse sentido, é de extrema importância a formulação de uma teoria geral de princípios de abstração que ofereça critérios claros para determinar quais instâncias de princípios de abstração são aceitáveis. ${ }^{14}$ É precisamente isso o que eu tinha

\footnotetext{
${ }^{13}$ Uma proposta análoga é oferecida por Heck (1993), tomando como base uma análise do Grundgesetze der Arithmetik.

14 Para uma análise do estatuto lógico de princípios de abstração e sua relação com o programa neologicista, cf. o artigo de WEIR (2003).
} 
em mente, quando mencionei, logo na introdução, que, do ponto de vista da argumentação lógica e filosófica, a realização de uma análise sistemática de princípios de abstração deve ser anterior à execução de qualquer programa de fundamentação da aritmética que pressuponha o uso de tais princípios.

É importante ressaltar que não faz parte do objetivo do presente artigo investigar se as propostas neologicistas tais como as apresentadas por Wright, Boolos e Heck, estão adequadas ao programa original fregeano, juntamente com todos seus pressupostos e consequências epistemológicas e ontológicas envolvendo noções como a de objetos lógicos, conceitos e extensóes defendidas por Frege. ${ }^{15}$ Trata-se aqui fundamentalmente de apresentar a necessidade e a relevância de uma teoria satisfatória sobre o estatuto lógico e filosófico dos princípios de abstração. É claro que, no contexto de fundamentação da aritmética, a partir dessa investigação, podemos derivar resultados úteis os quais podem revelar até que ponto tais princípios colaboram para a viabilidade formal do programa neologicista, além de mostrar quais as consequências filosóficas gerais dessa colaboraçáo.

\section{O IDEAL DE UMA TEORIA GERAL DE PRINCíPIOS DE ABSTRAÇÃo: ALGUMAS INDICAÇÓES DE PESQUISA}

Do modo como penso, a realização de uma análise de princípios de abstração está inevitavelmente ligada a alguns tópicos relevantes, tais como: a distinção de ordens lógicas de instâncias do princípio de abstração, o princípio do círculo vicioso, a noção de impredicatividade etc. Embora alguns desses tópicos, se tomados de maneira isolada, possam aparentemente apresentar um caráter excessivamente técnico e/ou definicional, sua análise é de extrema relevância para a formulação e discussão dos principais problemas com os quais os princípios de abstração estão comumente envolvidos, seja no âmbito mais restrito da fundamentação da aritmética, seja no âmbito mais geral dos problemas metafísicos e epistemológicos. Não por acaso, tais tópicos constituem o ponto de partida de qualquer análise acerca da consistência de procedimentos formais de abstração.

Primeiramente, uma análise de princípios de abstração deve ter como clara a ideia de que diferentes instâncias do esquema geral de abstração podem possuir diferentes ordens lógicas. Vejamos como isso ocorre. Como mostrei na

\footnotetext{
${ }^{15}$ Para uma análise da relação entre o programa logicista fregeano e a proposta neologicista, recomendo fortemente o artigo de RUFINNO (2003).
} 
Seção 1, a formalização do esquema geral de abstração pode ser dada como segue:

$$
\forall \mathrm{x} \forall \mathrm{y}(\Sigma \mathrm{x}=\Sigma \mathrm{y} \leftrightarrow \mathrm{x} \approx \mathrm{y})
$$

Nela, " $\Sigma$ " é um operador aplicável a expressôes do tipo das variáveis “x" e "y", e “ $\approx$ " caracteriza uma relação de equivalência entre entidades denotadas pelos termos que substituem as variáveis em questão. É fácil notar que podemos obter diferentes instâncias de princípios de abstração. Em geral, isso vai depender do tipo de termo que substitui as variáveis " $\mathrm{x}$ " e " $\mathrm{y}$ ". Caso as variáveis sejam substituídas por termos para indivíduos particulares - a exemplo dos termos singulares - o símbolo “ $\approx$ ” opera enquanto uma relaçáo diádica de primeira ordem, enquanto o princípio de abstração resultante representa uma abstração de primeira ordem sob objetos. No entanto, os casos mais interessantes - e, não por mera coincidência, também os casos mais polêmicos - de instâncias do princípio de abstração envolvem a substituição das variáveis " $\mathrm{x}$ " e " $\mathrm{y}$ " presentes na formalização acima por termos de ordem lógica superior no nível elementar dos objetos, tais como conceitos. ${ }^{16} \mathrm{Como}$ vimos, esse é precisamente o caso do Axioma V e do Princípio de Hume. O fato é que, como observa Fine (2002, p.1), instâncias de ordem superior do princípio de abstração “[...] envolvem 'projeçôes' de um largo domínio de conceitos em um domínio restrito de objetos, onde esses mesmos objetos podem eles mesmos cair sob os conceitos aos quais a abstração é feita”. É precisamente essa característica reflexiva de instâncias de ordem superior de princípios abstração que os torna, por um lado, extremamente poderosos, e, por outro, extremamente perigosos.

Até onde conheço o cenário atual da literatura sobre princípios de abstração, Kit Fine é o filósofo que mais fez avanços no sentido de formular uma teoria geral sobre abstração da maneira com que venho me referindo, ao longo deste artigo. Como Fine afirma em seu livro The Limits of Abstraction, a visão geral sobre o tema é que objetos abstratos devem ser tratados em termos de classes de equivalência e, na maioria das vezes, essa equivalência é concebida enquanto equivalência de conjuntos; sendo conjuntos entendidos aqui em termos de

\footnotetext{
${ }^{16}$ Kit Fine apresenta a distinção de ordem entre instâncias do princípio de abstração, através dos termos princípio de abstração objetual para abstraçôes de primeira ordem e princípio de abstração conceitual para abstraçóes de ordem superior (cf. FINE, 2002, p. 1). Nos Fundamentos da Aritmética de Frege, podemos encontrar exemplos de instâncias dos dois tipos: a abstraçấo que deriva a noção de direção de uma reta, a partir da noção de retas paralelas, constitui uma abstração objetual, ao passo que a abstraçáo que deriva a noção de equinumerosidade, com base na correspondência biunivoca entre elementos das extensóes de dois conceitos quaisquer - realizada no Princípio de Hume - é uma abstração conceitual.
} 
extensôes dos conceitos a partir dos quais os objetos são abstraídos. Com isso, Fine (2002, p.2) defende que o objetivo de uma teoria de princípios de abstração é tornar muito mais compreensiva a constituição e os limites das relações entre essas classes ou conjuntos e os conceitos envolvidos em procedimentos de abstração. Ignorar essas relações fundamentais é um passo na direção dos paradoxos. Ainda de acordo com Fine, a aparência inofensiva de instâncias de ordem superior do princípio de abstração, a exemplo do axioma $\mathrm{V}$, é, em grande parte, derivada da falsa ideia de que os conceitos são dados independentemente dos objetos que são abstraídos deles. Em linhas gerais, o argumento de Fine (2002, p.4) é que os critérios de identidade estabelecidos por princípios de abstração estão fundamentados numa relação de equivalência reflexiva, simétrica e transitiva entre classes. A sugestáo é que, para evitar paradoxos, esses critérios de identidade não devem ser inflacionários, ou seja, o número de classes de equivalência não deve ultrapassar o número de objetos envolvidos na abstração. Da mesma forma, esses critérios não podem ser circulares, quer dizer, pressupor a mesma noção de abstração que eles estão introduzindo.

Como ficou evidente na seção 1 , a polêmica criada no contexto da Filosofia da Matemática em torno do uso de instâncias de ordem superior do esquema geral de abstração deve-se, fundamentalmente, à derivação de alguns paradoxos com base em tais instâncias. Em linhas gerais, a derivação de paradoxos no contexto dos procedimentos técnicos de abstração está comumente associada ao uso, implícito ou explícito, de definiçóes impredicativas $^{17} \mathrm{e}$, consequentemente, a algum grau de desobediência do princípio do círculo vicioso. Basicamente, o que o princípio do círculo vicioso sustenta é que nenhuma totalidade pode conter elementos definíveis exclusivamente em termos dessa mesma totalidade. Em linhas gerais, a ideia que orienta todo esse debate é que, se princípios de abstração são usados na fundamentação da aritmética para introduzir uma definiçẫo contextual de número cardinal, juntamente com as condiçôes de verdade para sentenças numéricas, entâo o estabelecimento dessas mesmas condiçôes deveria ser não-circular, ou seja, a definição não deveria fazer uso do termo que está sendo definido. É precisamente a esse pré-requisito de não circularidade que as definiçôes impredicativas "embutidas" nas diferentes instâncias de ordem superior do esquema geral de abstração claramente não obedecem.

${ }^{17}$ Para uma apresentação geral da relaçấo entre impredicatividade e a Filosofia da Matemática, cf. FEFFERMAN (2007). 
Para tornar clara essa conexão entre procedimentos de abstração, o princípio do círculo vicioso e as definiçóes impredicativas, podemos tomar o Axioma $\mathrm{V}$ como um caso paradigmático. $\mathrm{O}$ Axioma $\mathrm{V}$ envolve certo tipo de circularidade viciosa; e foi precisamente essa circularidade que Russell explorou, na formulação do paradoxo que leva seu nome. O princípio do círculo vicioso foi definido no Principia Mathematica, de Russell e Whitehead, como parte da tentativa de oferecer critérios claros para evitar situações como a do Paradoxo de Russell, e estava intimamente ligado à formulação da chamada teoria ramificada dos tipos. É oportuno lembrar que, como vimos acima, a contradiçáo derivada por Russell a partir do Axioma $V$ postulava a existência de um conjunto definido intensionalmente, através da expressão "o conjunto de todos os conjuntos que não contêm a si mesmos como elementos”. É fácil observar que tal expressão fere o princípio do círculo vicioso e, portanto, não poderia ser legitimamente usada, o que evitaria assim o famoso paradoxo. Vale ressaltar que toda definição a qual fere o princípio do círculo vicioso é dita impredicativa.

No entanto, um elemento complicador para a discussão da relação entre impredicatividade e abstração é que nem sempre definiçôes impredicativas conduzem a uma contradição. Por exemplo, a expressão "o jogador mais alto do time", quando usada para definir um indivíduo, o faz de maneira impredicativa, pois, por meio dela definimos um indivíduo pressupondo a totalidade onde ele está inserido. Não obstante, até onde podemos perceber, dela não é possível derivar nenhuma situação paradoxal. Se há uma circularidade na definiçáo acima, ela é virtuosa. Portanto, do ponto de vista lógico, ela é uma expressão perfeitamente adequada e não problemática. Nesse sentido, o princípio do círculo vicioso parece ser excessivamente restritivo, quer dizer, ele proíbe não apenas definiçóes que levam a paradoxos, mas também definiçóes corriqueiras e não problemáticas, uma típica situação que se costuma caracterizar pela expressão “jogar fora a água do banho juntamente com o bebê". Os resultados recentes desenvolvidos pelos neologicistas mencionados na seção 2 foram expostos no sentido de provar a analiticidade do Princípio de Hume ${ }^{18}$ e, em linhas gerais, tendem a concordar que, embora ele envolva algum tipo de circularidade, é bastante razoável tomá-lo como consistente. O problema é oferecer um critério que diferencie boas e más definiçôes impredicativas.

Da forma como penso, toda essa discussão está ainda em aberto e longe de uma saída consensual. O que parece claro é que, para além dos impasses

${ }^{18}$ Cf. BOOLOS (1987, 1997), HECK (1993), dentre outros. 
apresentados acima, fica de positivo o fato de que temos boas razóes para pensar que uma solução para as questóes da impredicatividade e do princípio do círculo vicioso possa proporcionar bons insights para a caracterização e a identificação de instâncias do esquema geral de abstração livres de paradoxos. Talvez esse constitua um ponto de partida adequado para a formulação de uma teoria geral de princípios de abstração. Obviamente, a execução de uma teoria nos moldes que estou propondo aqui possui um elevado grau de sofisticação. Com isso, não faz parte da minha pretensão neste artigo traçar um esboço de tal teoria, apenas, como efetivamente fiz, mostrar sua relevância e indicar algumas diretrizes a partir das quais esse projeto possa ser executado.

PONTES, André Nascimento. A Proposal for a General Theory of Principles of Abstraction: a Contribution to the Foundation of Arithmetic. Trans/Form/Ação, Marília, v. 36, n. 2, p. 179-194, Maio/Ago., 2013.

\begin{abstract}
The objective of this paper is to present the correlation between fregean logicist program of the arithmetic foundation and Crispin Wright's neologicism and the so-called abstraction principles. My theses is that a general analyses of the abstraction principles, from a logical and explanatory point of view, is more basic than the project of the arithmetic foundation in the way that it was proposed by the logicism. That became evident through the fact that the failure in the logicist program was closely connected to imprecision and to inconsistency in the use of abstraction procedures.
\end{abstract}

KEYWORDS: Logic. Philosophy of Mathematics. Arithmetic. Abstraction Principle.

\title{
REFERÊNCIAS
}

BOOLOS, George. Is Hume's Principle Analytic? In: HECK, R. (Ed.). Language, Thought and Logic: Essays in honour of Michael Dummett. Oxford: Oxford University Press, 1997.

The Consistency of Frege's Foundations of Arithmetic. In: DEMOPOULOS, W. (Ed.). Fregés philosophy of mathematics. Cambridge: Harvard University Press, 1995, p. 211-233. [Publicado pela primeira vez em 1987]

DEMOPOULOS, William (Ed.). Freges philosophy of mathematics. Cambridge: Harvard University Press, 1995. 
; CLARK, P. The logicism of Frege, Dedekind and Russell. In: SHAPIRO, S. (Ed.). The Oxford Handbook of Philosophy of Mathematics and Logic. Oxford: Oxford University Press, 2007, p. 129-165.

FEFERMAN, Salomon. Predicativity. In: SHAPIRO, S. (Ed.). The Oxford Handbook of Philosophy of Mathematics and Logic. Oxford: Oxford University Press, 2007, p. 590-624.

FINE, Kit. The Limits of abstraction. Oxford: Clarendon Press, 2002.

FREGE, Gottlob. Fundamentos da Aritmética. São Paulo: Abril Cultural, 1983 (Col. Os Pensadores).

GREIMANN, Dirk. What is Frege's Julius Caesar Problem? Dialectica, vol. 57, n. 3, p. 261-278, 2003.

HALE, B.; WRIGHT, C. Logicism in the twenty-first century. In: SHAPIRO, S. (Ed.). The Oxford Handbook of Philosophy of Mathematics and Logic. Oxford: Oxford University Press, 2007, p. 166-202.

. The Reason's Proper Study: essays towards a neo-fregean philosophy of mathematics. Oxford: Oxford University Press, 2001.

HECK JUNIOR, Richard. The development of Arithmetic in Frege's Grundgesetze der Arithmetik. The Journal of Symbolic Logic, 58, n. 2, p. 579-601, 1993.

. Frege's Theorem: an introduction. Manuscrito, Vol. 26, n.2, p. 471-503, Jul.-Dez. de 2003 [Publicado pela primeira vez em 1998]

PARSONS, Charles. On the consistency of the first-order portion of Frege's logical system. In: DEMOPOULOS, W. (Ed.). Frege's philosophy of mathematics. Cambridge: Harvard University Press, 1995, p. 442-431. [Publicado pela primeira vez em 1987]

RUFFINO, Marco. Why Frege would not be a Neo-fregean. Mind, Vol. 112, p. 51-78, jan. de 2003.

WEIR, Alan. Neo-Fregeanism: an embarrassment of riches. Notre Dame Journal of Formal Logic. Vol. 44, n. 1, 2003.

WRIGHT, Crispin. Frege's conception of numbers as objects. Aberdeen: Aberdeen University Press, 1983.

Recebido em: 25.05.2012

Aceito em: 18.12.2012 